\title{
Role of P14 and MGMT Gene Methylation in Hepatocellular Carcinomas: a Meta-analysis
}

\author{
Cheng-Cheng $\mathrm{Li}^{1}$, Zhuang $\mathrm{Yu}^{2}$, Lian-Hua Cui ${ }^{1 *}$,Jin-Mei Piao', Meng Liu ${ }^{1}$
}

\begin{abstract}
Background: This meta-analysis was performed to investigate the relationship between methylation of the P14 and O6-methylguanine-DNA methyltransferase (MGMT) genes and the risk of hepatocellular carcinoma (HCC). Materials and Methods: We searched PubMed, EMBASE, the Chinese Biomedical Database (CBM), and the China National Knowledge Infrastructure (CNKI) databases to identify relevant studies that analysed HCC tissues for P14 and MGMT gene methylation status; we then performed a meta-analysis. Odds ratios (ORs) and $95 \%$ confidence intervals $(95 \% \mathrm{CIs})$ were calculated to evaluate the association between gene methylation and the risk of HCC. Results: Ten studies that assessed P14 gene methylation in $630 \mathrm{HCC}$ tumour tissues and nine studies analysing MGMT methylation in $497 \mathrm{HCC}$ tumour tissues met our inclusion criteria. Our meta-analysis revealed that the rate of P14 methylation was significantly higher in HCCs than in adjacent tissues (OR 3.69, 95\% CI 1.63-8.35, $p=0.002$ ), but there was no significant difference in MGMT methylation between HCC and adjacent tissues (OR 1.76, 95\% CI $0.55-5.64, p=0.34)$. A subgroup analysis according to ethnicity revealed that P14 methylation was closely related to the risk of HCC in Chinese and Western individuals (Chinese, OR 7.74, 95\% CI 1.36-44.04, $p=0.021$; Western, OR 3.60, 95\% CI 1.49-8.69, $p=0.004)$. Furthermore, MGMT methylation was not correlated with the risk of $\mathrm{HCC}$ in Chinese individuals (OR 2.42, 95\% CI 0.76-7.73, $p=0.134)$. The combined rate of P14 methylation was $35 \%$ (95\% CI 24-48\%) in HCC tumour tissues and $11 \%(95 \%$ CI 4-27\%) in adjacent tissues, whereas the combined rate of MGMT methylation was $15 \%(95 \% \mathrm{CI}$ 6-32\%) in HCC and $10 \%(95 \% \mathrm{CI} 4-22 \%)$ in adjacent tissues. Conclusions: These results suggest that the risk of HCC is related to P14 methylation, but not MGMT methylation. Therefore, P14 gene methylation may be a potential biomarker for the diagnosis of $\mathrm{HCC}$.
\end{abstract}

Keywords: Methylation - P14 - MGMT - hepatocellular carcinoma - meta-analysis

Asian Pac J Cancer Prev, 15 (16), 6591-6596

\section{Introduction}

Hepatocellular carcinoma (HCC) is one of the most frequent malignancies and the third leading cause of cancer-related deaths worldwide (Iakova et al., 2011). However, the molecular and cellular mechanisms of hepatocellular carcinoma pathogenesis remain unclear. Previous studies have suggested that aberrant methylation of tumour suppressor genes, which results in gene inactivation, may play a role in the development of HCC (Moribe et al., 2009).

$\mathrm{P} 14$ and $\mathrm{O}^{6}$-methylguanine-DNA methyltransferase (MGMT) are two common tumour suppressor genes. The P14 ${ }^{\mathrm{ARF}}$ gene is located on chromosome $9 \mathrm{p} 21$, and its gene product regulates one of the most important cell cycle regulatory pathways, which also plays a key role in carcinogenesis (Zhang et al., 1998). The P14 protein stabilises cellular p53 levels by combining with and accelerating the degradation of murine double minute 2 (MDM2). This enhances the p53 restriction point effect in the G1 to $\mathrm{S}$ and $\mathrm{G} 2$ to $\mathrm{M}$ phase transitions of the cell cycle and finally arrests cell division in G1 phase to induce apoptosis (Pomerantz et al., 1998). MGMT is a DNA repair gene that recovers the activity of injured guanine by removing alkyl adducts from the $\mathrm{O}^{6}$ position, protecting cells from the carcinogenic effects of adducts (Ishikawa et al., 2004; Esteller, 2006). In recent years, the loss of P14 and MGMT expression and their aberrant gene methylation have been reported in the tumour tissues of various human neoplasias, including HCC (Esteller et al., 1999; Zhang et al., 2008). Therefore, P14 and MGMT methylation may be useful biomarkers for the diagnosis of HCC.

Although several recent studies have assessed the association of P14 and MGMT methylation with the risk of HCC, the data are highly diverse due to differences among studies, including different ethnicities, small sample sizes, and differences in detection methods. The range of P14 and MGMT methylation in HCC tissues was $0-80 \%$ and $0-64.3 \%$ (Tannapfel et al., 2001; Yu et al., 2002; Yang et al., 2003; Yu et al., 2003; Anzola et al., 2004; Qian et al., 2005; Liu et al., 2006; Nomoto et al., 2007; Ruan et al., 2007; Lou et al., 2008; Herath et al., 2009; Li et al., 2010; Li et al., 2013), respectively, and 
some studies reported that the levels of methylation were higher in control than in cancer tissues (Su et al., 2007; Zhang et al., 2007; Formeister et al., 2010). Based on these inconsistent results, we performed a meta-analysis using the accumulated data to accurately assess the association between P14 and MGMT methylation and the risk of HCC.

\section{Materials and Methods}

\section{Primary Search Strategy}

We conducted a comprehensive search of the related studies using PubMed, EMBASE, the Chinese Biomedical Database (CBM) and the China National Knowledge Infrastructure (CNKI) databases up to March 2014 regarding the relationship between methylation of the P14 and MGMT genes and the risk of HCC. The following keywords used were: "HCC," "liver cancer," "P14," "P14ARF," "MGMT," "O'-methylguanine-DNA methyltransferase" and "methylation." The language in which the articles published was restricted to English and Chinese. Moreover, the references cited in the publications were conducted to scan for additional relevant studies.

\section{Inclusion and Exclusion Criteria}

Studies which were eligible for inclusion could meet the following criteria : (1) they were case-control studies regarding the correlation between methylation of P14 and MGMT genes and the risk of HCC. (2) they were performed in the HCC patient. (3) P14 and MGMT gene methylation status were detected by using methylationspecific PCR (MSP) or real-time quantitative MSP (QMSP). (4) specimens of HCC must be surgically removed tumor tissues and the control groups were composed of adjacent or other non-tumor tissues. (5) the number of samples was more than 10. (6) these studies must include sufficient data about rate of P14 and MGMT methylation.

\section{Data Extraction}

Data extraction was systematically performed by two reviewers using standardized data extraction forms. For each study, the following information was extracted: first author, year of publication, country, sample size, detection method and the rate of gene methylation. The discrepancy was resolved by discussion.

\section{Methodological Assessment}

The Newcastle-Ottawa Scale (NOS) criteria was conducted to assess the methodological quality of each included study (Stang, 2010). It consisted of three aspects: (1) subject selection 0-4; (2) comparability of subject 0-2; (3) clinical outcome $0-3$. NOS scores fluctuated from 0 to 9 with a score $\geq 7$ identified a good quality.

\section{Statistical Analysis}

The pooled ORs and its corresponding 95\%CI were calculated to evaluate the association between methylation of P14 and MGMT genes and the risk of HCC. The significance of the pooled ORs was evaluated by using the $\mathrm{Z}$ test, and $p<0.05$ was considered as statistically significant. A chi-square-based $\mathrm{Q}$ and $\mathrm{I}^{2}$ test were used to evaluate the heterogeneity among included studies (Higgins; Thompson, 2002). If Q test reveals a $p<0.05$ or $\mathrm{I}^{2}$ test shows $>50 \%$ which identified significant heterogeneity, the random-effects model (the inverse variance method) was used to assess the pooled ORs; otherwise, the fixed-effects model (the Mantel-Haenszel method) was applied (Fleiss, 1993). Subgroup analysis was performed according to ethnicity. Furthermore, we conducted to calculate the combined rate of P14 and MGMT methylation in HCC and adjacent tissues. The Begg's Funnel plot and Egger's test were conducted to investigate publication bias, and $p<0.05$ was considered as statistically significant publication bias (Egger et al., 1997). In addition, we performed sensitivity analysis to evaluate whether the results were influenced, when removed one study at a time. The pooled ORs and its corresponding 95\%CI were performed by STATA 12.0 software (Stata corp., College Station, TX). The combined rate of P14 and MGMT methylation were conducted by $\mathrm{R}$ 3.0.3 software (Luo et al., 2013). All $p$ values were twotailed, and $p<0.05$ was statistical significance.

\section{Results}

\section{Study Characteristics}

Ten studies that assessed P14 gene methylation in 630 HCC tumour tissues and nine studies analysing MGMT methylation in 497 HCC tumour tissues met our inclusion criteria. Methylation-specific polymerase chain reaction (MSP) was conducted in all included studies. NOS scores of these studies were $\geq 5$. The study characteristics and methodological quality regarding P14 and MGMT methylation were summarized in (Table 1 and Table 2), respectively.

P14 gene methylation in HCC tumor tissues and adjacent tissues

Ten studies included 630 HCC tumor tissues and 517 adjacent tissues concentrated on the association between P14 methylation and the risk of HCC. As shown in (Figure $1)$, there was significant heterogeneity among these studies $\left(p=0.002, \mathrm{I}^{2}=66.7 \%\right)$, which analyzed using the randomeffects model. The meta-analysis indicated that the rate of P14 methylation was significantly higher in HCC than in adjacent tissues (OR 3.69, 95\% CI 1.63-8.35, $p<0.05$ ).

MGMT gene methylation in HCC tumor tissues and adjacent tissues

Similarly, nine studies with 497 HCC tumor tissues and 407 adjacent tissues focused on the relationship between MGMT methylation and the risk of HCC. As heterogeneity also existed among these studies $\left(p<0.001, \mathrm{I}^{2}=78.0 \%\right)$, the random-effects model was used to calculate the pooled OR (Figure 2). The meta-analysis indicated that, however, there was no significant different in MGMT methylation between HCC and adjacent tissues (OR 1.76, 95\%CI $0.55-5.64, p=0.338)$.

\section{Subgroup analysis}

The random-effects model was performed due to significant heterogeneity among these studies (P14: 
Table 1. Characteristics of the Included Studies (P14) in Meta-Analysis

\begin{tabular}{|c|c|c|c|c|c|c|c|}
\hline First author and Year & County & $\mathrm{HCC}$ tissue/control & Number & Age (years) & $\operatorname{Sex}(M / F)$ & Method & $\begin{array}{l}\text { NOS } \\
\text { score }\end{array}$ \\
\hline Li et al (2013). & China & Tumour/adjacent/cirrhotic tissues & $44 / 44 / 41$ & No data & No data & MSP & 5 \\
\hline Li et al (2010). & China & Tumour/adjacent tissues & $115 / 48$ & $50(27-76)$ & $84 / 31$ & MSP & 7 \\
\hline Herath et al (2009). & $\begin{array}{l}\text { Australian/ } \\
\text { South Africa }\end{array}$ & $\begin{array}{l}\text { Tumour/adjacent/ } \\
\text { normal liver tissues }\end{array}$ & $61 / 61 / 20$ & No data & $52 / 9$ & MSP & 6 \\
\hline Ruan et al (2007). & China & Tumour/adjacent tissues & $42 / 42$ & No data & No data & MSP & 5 \\
\hline Zhang et al (2007). & China & Tumour/adjacent tissues & $50 / 50$ & 51.6 & $44 / 6$ & MSP & 7 \\
\hline Liu et al (2006). & China & Tumour/adjacent tissues & $50 / 48$ & $48.5(28-76)$ & $44 / 6$ & MSP & 7 \\
\hline Anzola et al (2004). & Spain & Tumour/adjacent tissues & $117 / 110$ & No data & $58 / 20$ & MSP & 6 \\
\hline Yang et al (2003). & America & Tumour/adjacent/cirrhotic tissues & $51 / 14 / 15$ & $58(27-81)$ & $30 / 21$ & MSP & 7 \\
\hline Yu et al (2002). & China & Tumour/adjacent tissues & $29 / 29$ & 49.55 & $23 / 6$ & MSP & 7 \\
\hline Tannapfel et al (2001). & Germany & Tumour/adjacent tissues & $71 / 71$ & No data & No data & MSP & 5 \\
\hline
\end{tabular}

*M, male; F, female; NOS, Newcastle-Ottawa Scale; MSP, methylation-specific polymerase chain reaction

Table 2. Characteristics of the included studies (MGMT) in meta-analysis

\begin{tabular}{|c|c|c|c|c|c|c|c|}
\hline First author and Year & County & $\mathrm{HCC}$ tissue/control & Number & Age (years) & $\operatorname{Sex}(\mathrm{M} / \mathrm{F})$ & Method & $\begin{array}{l}\text { NOS } \\
\text { score }\end{array}$ \\
\hline Formeister et al. (2010) & America & Tumour/adjacent tissues & $43 / 45$ & 66.2 & No data & MSP & 6 \\
\hline Li et al. (2010) & China & Tumour/adjacent tissues & $115 / 48$ & $50(27-76)$ & $84 / 31$ & MSP & 7 \\
\hline Herath et al. (2009) & Australian & Tumor/adjacent/normal liver tissues & $61 / 61 / 20$ & No data & $52 / 9$ & MSP & 6 \\
\hline Lou et al. (2008) & China & Tumour/adjacent/cirrhotic tissues & $60 / 60 / 16$ & $53.3(22-75)$ & $51 / 9$ & MSP & 7 \\
\hline Nomoto et al. (2007) & Japan & Tumour/adjacent/normal liver tissues & $42 / 19 / 17$ & 59.26 & No data & MSP & 6 \\
\hline Su et al. (2007) & China & Tumour/adjacent tissues & $58 / 58$ & $56.2(29-85)$ & $54 / 4$ & MSP & 7 \\
\hline Liu et al. (2006) & China & Tumour/adjacent tissues & $50 / 48$ & $48.5(28-76)$ & $44 / 6$ & MSP & 7 \\
\hline Qian et al. (2005) & China & Tumour/adjacent tissues & $40 / 40$ & 50.3 & $30 / 10$ & MSP & 7 \\
\hline Yu et al. (2003) & China & Tumour/adjacent tissues & $28 / 28$ & No data & No data & MSP & 5 \\
\hline
\end{tabular}

*M, male; F, female; NOS, Newcastle - Ottawa Scale; MSP, methylation - specific polymerase chain reaction

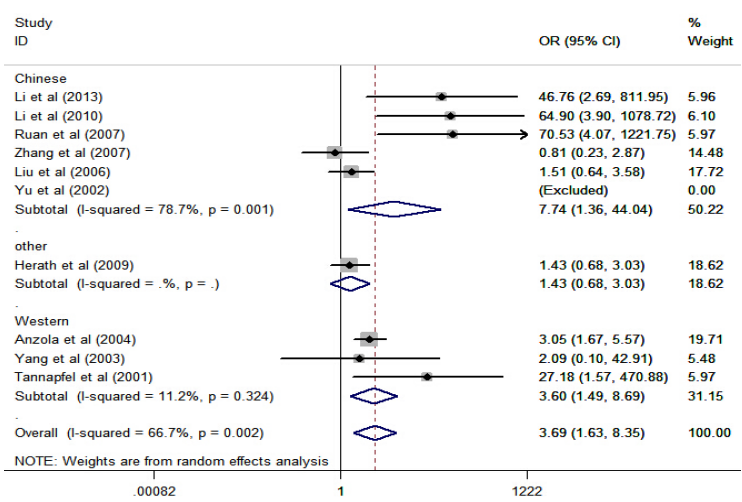

Figure 1. Forest Plot for the Association Between P14 Methylation and the Risk of HCC

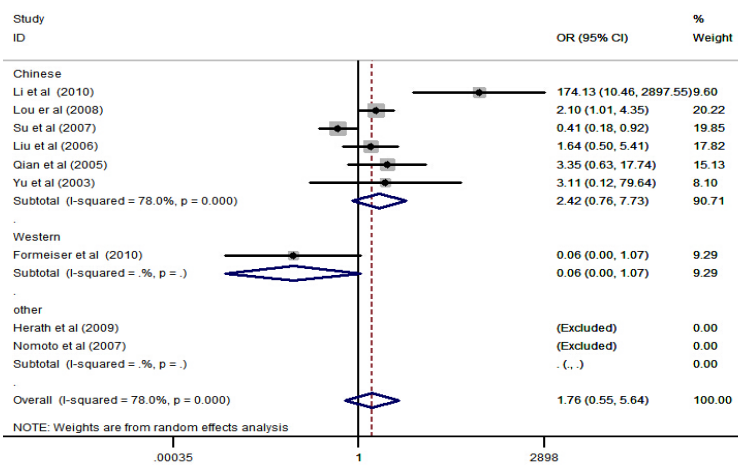

Figure 2. Forest Plot for the Association Between MGMT Methylation and the Risk of HCC

$\mathrm{I}^{2}=66.7 \%$, MGMT: $\mathrm{I}^{2}=78 \%$ ). Further subgroup analysis according to ethnicity was performed. The subgroup metaanalysis indicated that $\mathrm{P} 14$ methylation was closely related to the risk of HCC in Chinese and Western individuals (Chinese, OR 7.74,95\%CI 1.36-44.04, $p=0.021$; Western, OR 3.60, 95\%CI 1.49-8.69, $p=0.004)$, which showed a

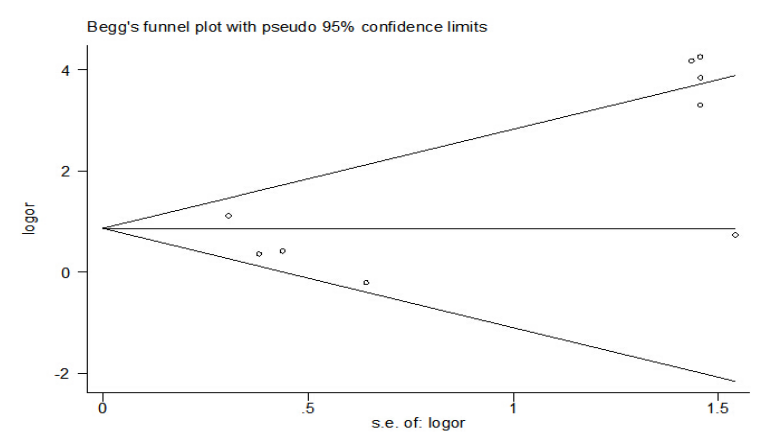

Figure 3. Begg's Funnel Plot of Publication Biases on the Relationship Between P14 Methylation and the Risk of HCC

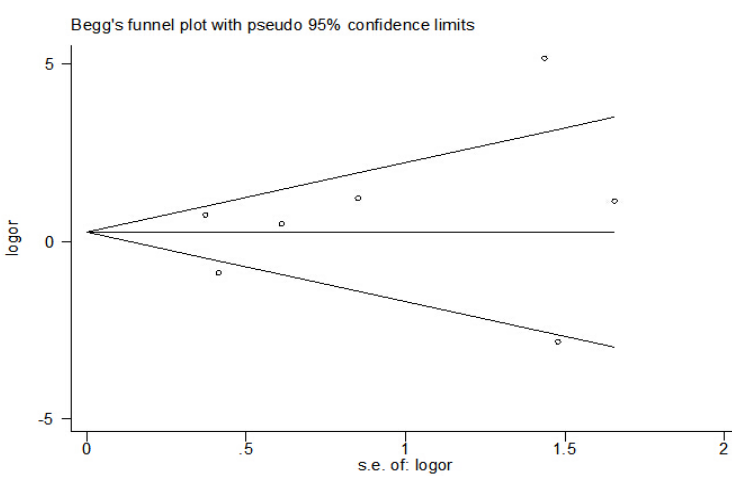

Figure 4. Begg's Funnel Plot of Publication Biases on the Relation Between MGMT Methylation and the Risk of HCC

stronger relationship in Chinese than in Western patients (Figure 1). Similarly, a subgroup meta-analysis also revealed that MGMT methylation was not correlated with HCC risk in Chinese subjects (OR 2.42, 95\%CI 0.76-7.73, $p=0.134$ ) (Figure 2). 


\section{Combined rate of $P 14$ and MGMT methylation}

As there was significant heterogeneity among these studies (all $\mathrm{I}^{2}>50 \%, p<0.05$ ), the random-effects model was used to assess the combined rate of P14 and MGMT methylation. Our meta-analysis revealed that the combined rate of P14 methylation was 35\% (95\% CI $24-48 \%$ ) in HCC tumor tissues and 11\% (95\%CI 4-27\%) in adjacent tissues. Similarly, our meta-analysis indicated that the combined rate of MGMT methylation was $15 \%$ (95\% CI 6-32\%) in $\mathrm{HCC}$ tumor tissues and 10\% (95\% CI 4-22\%) in adjacent tissues.

\section{Publication bias and Sensitivity analysis}

We conducted Begg's funnel plot and Egger's test to detect the publication bias of included studies. As shown in (Figure 3 and Figure 4), although the Begg's funnel plots revealed a little asymmetry, Egger's tests which offered specific data showed no obvious Publication bias (all $p>0.05$ ). Sensitivity analysis showed that the pooled ORs did not influence by eliminating one study at a time, indicating that our conclusion were robust.

\section{Discussion}

Aberrant methylation of tumour suppressor genes, which can cause transcriptional inhibition and gene inactivity (Citron et al., 1991), is an important pathway in the formation and progression of various human cancers. Thus, gene methylation status may be a potential new biomarker to assess tumour risk as well as to achieve early diagnosis and evaluate prognosis (Kim et al., 2005; Saelee et al., 2012).

Some studies have reported that P14 and MGMT gene methylation are relatively common in HCC (Yang et al., 2003; Yu et al., 2003; Ael-R et al., 2013). However, the data are highly diverse due to differences among studies, including different ethnicities, small sample sizes, and different detection methods. Therefore, we performed a meta-analysis using the accumulated data to evaluate the relationship between P14 and MGMT methylation and the risk of HCC.

Based on the inclusion and exclusion criteria, ten studies analysing P14 and nine assessing the correlation between MGMT methylation and the risk of HCC were included. The rate of gene methylation for P14 ranged from 6 to $88 \%$; eight studies reported that the gene methylation rate was higher in HCC than in adjacent tissues. Additionally, one study found that P14 methylation was $0 \%$ in both HCC tumour and adjacent tissues, and one found that P14 methylation was higher in adjacent tissues than in HCC. Our meta-analysis revealed that the overall P14 methylation rate was 35\% (95\%CI 24$48 \%$ ) in HCC tumour tissues vs. $11 \%$ (95\% CI 4-27\%) in adjacent tissues. The pooled OR was 3.69 (95\% CI 1.63-8.35), which suggests that P14 gene methylation was associated with a risk of HCC. P14 methylation has also been reported in other cancers (Tian et al., 2006; He et al, 2011). For example, Tian et al. (2006) reported that the P14 methylation rate was $17.5 \%$ in non-small-cell lung cancer tumour tissues and $2.5 \%$ in adjacent tissues. Similarly, He et al. (2011) found that the rate of P14 methylation in glioma tissues was $39.4 \%$ vs. $0 \%$ in non-cancer tissues. These data suggest that $\mathrm{P} 14$ gene methylation may play an important role in cancer development.

We also analysed nine studies that assessed the association between MGMT methylation and the risk of HCC. Although five studies found a higher rate of MGMT methylation rate in HCC tumour tissues than in adjacent tissues, the rate of methylation varied from 3.6 to $64.3 \%$. Two additional studies reported that the gene methylation rate was $0 \%$ in both HCC tumour and adjacent tissues, and one reported that $\mathrm{P} 14$ methylation was higher in adjacent tissues than in HCC tumour tissues. Our meta-analysis demonstrated that the combined rate of MGMT gene methylation was $15 \%$ (95\%CI 6-32\%) in HCC tumour tissues and $10 \%(95 \% \mathrm{CI} 4-22 \%)$ in the adjacent tissues. There was no significant association between MGMT methylation and the risk of HCC (OR 1.76, 95\%CI $0.55-5.64)$. Therefore, it remains controversial whether MGMT methylation is related to cancer risk. Gu et al. (2013) performed a meta-analysis that identified a strong relationship between MGMT methylation and the risk of non-small-cell lung cancer, with MGMT methylation much higher in cancer than in non-cancer tissues. In contrast, Kupcinskaite-Noreikiene et al. (2013) reported that MGMT methylation was lower in gastric carcinoma than in adjacent tissues. Moreover, Laing et al. (2010) found no relationship between MGMT methylation and cutaneous squamous cell carcinoma $(p=0.21)$.

We also performed stratified analyses according to ethnicity. The subgroup meta-analysis revealed that P14 methylation was closely associated with the risk of HCC in Chinese and Western individuals (Chinese, OR 7.74, 95\%CI 1.36-44.04; Western, OR 3.60, 95\%CI 1.498.69). The relationship was stronger in Chinese than in Western patients because most studies included in this meta-analysis were performed in China, which has a high prevalence of hepatitis B virus (HBV)-related HCC compared with Western countries (Jemal et al., 2011). Additionally, P14 methylation was closely related to HBV infection (Zhang et al., 2014). Because too few studies assessed the MGMT methylation in Western populations, we only conducted a subgroup meta-analysis on studies with Chinese subjects. The results revealed that MGMT methylation was not correlated with $\mathrm{HCC}$ risk (OR 2.42, 95\%CI 0.76-7.73), consistent with the unstratified OR (OR 1.76, 95\%CI 0.55-5.64).

Sensitivity analysis was performed to investigate the effects of a single study on the combined OR. Studies were removed from the analyses one at a time, and the effect of study on the overall meta-analysis was assessed. No changes occurred, indicating that the results were robust. Additionally, publication bias was analysed using Begg's and Egger's tests, and no significant publication bias was identified.

Our meta-analysis has several potential limitations that must be acknowledged. First, selection bias was inevitable because the search strategy was limited to articles published in English or Chinese. Second, the study included only published studies, and some literature was unavailable. Therefore, potential publication bias should be considered. Third, we did not assess differences in 
P14 and MGMT methylation between tumour and benign tissues because of an insufficient number of relevant studies. Nevertheless, this is the first meta-analysis assessing the association between P14 and MGMT methylation and the risk of HCC.

In conclusion, the risk of HCC was related to P14 methylation, but not MGMT methylation. Therefore, P14 gene methylation may be a potential biomarker for the diagnosis of HCC.

\section{References}

Ael-R Z, Nassar AA, El-Din EMN, et al (2013). Disease progression from chronic hepatitis $\mathrm{C}$ to cirrhosis and hepatocellular carcinoma is associated with increasing DNA promoter methylation. Asian Pac J Cancer Prev, 14, 6721-6.

Anzola M, Cuevas N, Lopez-Martinez M, et al (2004). P14ARF gene alterations in human hepatocellular carcinoma. Eur J Gastroenterol Hepatol, 16, 19-26.

Citron M, Decker R, Chen S, et al (1991). O'-methylguanineDNA methyltransferase in human normal and tumor tissue from brain, lung, and ovary. Cancer Res, 51, 4131-4.

Egger M, Davey SG, Schneider M, Minder C (1997). Bias in meta-analysis detected by a simple, graphical test. $B M J$, 315, 629-34.

Esteller M (2006). Epigenetics provides a new generation of oncogenes and tumour-suppressor genes. Br J Cancer, 94, 179-83.

Esteller M, Hamilton SR, Burger PC, et al (1999). Inactivation of the DNA repair gene $\mathrm{O}^{6}$-methylguanine-DNA methyltransferase by promoter hypermethylation is a common event in primary human neoplasia. Cancer Res, 59, 793-7.

Fleiss JL (1993). The statistical basis of meta-analysis. Stat Methods Med Res, 2, 121-45.

Formeister EJ, Tsuchiya M, Fujii H, et al (2010). Comparative analysis of promoter methylation and gene expression endpoints between tumorous and non-tumorous tissues from $\mathrm{HCV}$-positive patients with hepatocellular carcinoma. Mutat Res, 692, 26-33.

Gu C, Lu J, Cui T, et al (2013). Association between MGMT promoter methylation and non-small cell lung cancer: a meta-analysis. PLoS One, 8 , e7263.

He J, Qiao JB, Zhu H (2011). p14ARF promoter region methylation as a marker for gliomas diagnosis. Med Oncol, 28, 1218-24.

Herath NI, Purdie DM, Kew MC, et al (2009). Varying etiologies lead to different molecular changes in Australian and South African hepatocellular carcinomas. Int J Oncol, 35, 1081-9.

Higgins JP, Thompson SG (2002). Quantifying heterogeneity in a meta-analysis. Stat Med, 21, 1539-58.

Iakova P, Timchenko L, Timchenko NA (2011). Intracellular signaling and hepatocellular carcinoma. Semin Cancer Biol, 21, 28-34.

Ishikawa T, Zhang SS, Qin X, et al (2004). DNA repair and cancer: lessons from mutant mouse models. Cancer Sci, 95, 112-7.

Jemal A, Bray F, Center MM, et al (2011). Global cancer statistics. CA Cancer J Clin, 61, 69-90.

Kim YT, Lee SH, Sung SW, Kim JH (2005). Can aberrant promoter hypermethylation of $\mathrm{CpG}$ islands predict the clinical outcome of non-small cell lung cancer after curative resection. Ann Thorac Surg, 79, 1180-8.

Kupcinskaite-Noreikiene R, Skieceviciene J, Jonaitis L, et al (2013). CpG island methylation of the MLH1, MGMT,
DAPK, and CASP8 genes in cancerous and adjacent noncancerous stomach tissues. Medicina, 49, 361-6.

Laing ME, Cummins R, O'Grady A, et al (2010). Aberrant DNA methylation associated with MTHFR C677T genetic polymorphism in cutaneous squamous cell carcinoma in renal transplant patients. Br J Dermatol, 163, 345-52.

Li B, Liu W, Wang L, et al (2010). CpG island methylator phenotype associated with tumor recurrence in tumor-nodemetastasis stage I hepatocellular carcinoma. Ann Surg Oncol, 17, 1917-26.

Li H, Gao B, Yu Z, Liu J, Zhang J (2013). Significance of detection of mutigene methylation in liver cancer tissues for early diagnosis of liver cancer. J Clin Hepatol, 29, 624-6.

Liu W, Wang L, Wang J, et al (2006). Correlations of CpG island methylator phenotype and OPCML gene methylation to carcinogenesis of hepatocellular carcinoma. Ai Zheng, 25, 696-700.

Lou C, Yang B, Gao Y, et al (2008). Aberrant methylation of multiple genes and its clinical implication in hepatocellular carcinoma. Zhonghua Zhong Liu Za Zhi, 30, 831-6.

Luo M, Tang H, Zhou Q, et al (2013). Realizing the meta-analysis of single rate in R software. J Evidence-based Medicine, 13, 181-8.

Moribe T, Iizuka N, Miura T, et al (2009). Methylation of multiple genes as molecular markers for diagnosis of a small, well-differentiated hepatocellular carcinoma. Int $J$ Cancer, 125, 388-97.

Nomoto S, Kinoshita T, Kato K, et al (2007). Hypermethylation of multiple genes as clonal markers in multicentric hepatocellular carcinoma. Br J Cancer, 97, 1260-5.

Pomerantz J, Schreiber-Agus N, Liegeois NJ, et al (1998). The Ink4a tumor suppressor gene product, p19Arf, interacts with MDM2 and neutralizes MDM2's inhibition of p53. Cell, 92, 713-23.

Qian B, Zhu L, Geng X, et al (2005). Methylation of MGMT, DAPK, THBS1 and RIZ1 genes in hepatocellular carcinoma. Chin J Gen Surg, 20, 291-4.

Ruan X, Wang S, Chen X, Ren P (2007). 5' CpG methylation of P14ARF gene in hepatocellular carcinoma. Chin J Gen Surg, 22, 848-50.

Saelee P, Chuensumran U, Wongkham S, et al (2012). Hypermethylation of suppressor of cytokine signaling 1 in hepatocellular carcinoma patients. Asian Pac J Cancer Prev, 13, 3489-93.

Stang A (2010). Critical evaluation of the Newcastle-Ottawa scale for the assessment of the quality of nonrandomized studies in meta-analyses. Eur J Epidemiol, 25, 603-5.

Su PF, Lee TC, Lin PJ, et al (2007). Differential DNA methylation associated with hepatitis B virus infection in hepatocellular carcinoma. Int J Cancer, 121, 1257-64.

Tannapfel A, Busse C, Weinans L, et al (2001). INK4a-ARF alterations and $\mathrm{p} 53$ mutations in hepatocellular carcinomas. Oncogene, 20, 7104-9.

Tian K, Lin L, Jia T, Guo X, Zhang L (2006). Promoter methylation status and protein expression of p14ARF gene in squamous cell carcinoma and adenocarcinoma of the lung. Zhongguo Fei Ai Za Zhi, 9, 40-44.

Yang B, Guo M, Herman JG, Clark DP (2003). Aberrant promoter methylation profiles of tumor suppressor genes in hepatocellular carcinoma. Am J Pathol, 163, 1101-7.

Yu J, Ni M, Xu J, et al (2002). Methylation profiling of twenty promoter-CpG islands of genes which may contribute to hepatocellular carcinogenesis. BMC Cancer, 2, 29.

Yu J, Zhang HY, Ma ZZ, et al (2003). Methylation profiling of twenty four genes and the concordant methylation behaviours of nineteen genes that may contribute to hepatocellular carcinogenesis. Cell Res, 13, 319-33. 
Cheng-Cheng Li et al

Zhang C, Guo X, Zhang L, et al (2008). Methylation-related silencing of p14ARF gene correlates with telomerase activity and mRNA expression of human telomerase reverse transcriptase in hepatocellular carcinoma. J Surg Oncol, 98, 462-8.

Zhang C, Li Z, Cheng Y, et al (2007). CpG island methylator phenotype association with elevated serum alpha-fetoprotein level in hepatocellular carcinoma. Clin Cancer Res, 13, 944-52.

Zhang JC, Gao B, Yu ZT, et al (2014). Promoter hypermethylation of p14 (ARF) , RB, and INK4 gene family in hepatocellular carcinoma with hepatitis B virus infection. Tumour Biol, 35, 2795-802.

Zhang Y, Xiong Y, Yarbrough WG (1998). ARF promotes MDM2 degradation and stabilizes p53: ARF-INK4a locus deletion impairs both the $\mathrm{Rb}$ and $\mathrm{p} 53$ tumor suppression pathways. Cell, 92, 725-34. 